\title{
Formation of brown dwarfs and planets
}

\author{
Åke Nordlund ${ }^{1}$ \\ ${ }^{1}$ Centre for Star and Planet Formation and Niels Bohr International Academy, \\ University of Copenhagen, Blegdamsvej 17, 2100 Copenhagen, Denmark \\ email: aake@nbi.dk
}

\begin{abstract}
Brown dwarfs and massive planets have similar structures, and there is probably an overlap in mass between the most massive planets and the lowest mass brown dwarfs. This raises questions as to what extent the structures of the most massive planets and lowest mass brown dwarfs differ, and what similarities (or not) there might be between their formation mechanisms. Here I discuss these issues on the background of recent numerical simulations of star formation, new evidence from cosmochemistry about the conditions in the early solar system, and recently discovered mechanisms that can expedite planetesimal and possibly planet formation greatly.
\end{abstract}

Keywords. stars: formation, planetary systems: formation

\section{Introduction}

Due to the properties of gas at the low temperature and high densities that characterize the interiors of massive planets and low mass stars their typical radii are similar and do not vary much with mass (Chabrier et al. 2000; Baraffe et al. 2002; Chabrier et al. 2009), and in this respect their interior structures are quite similar. It is thus fair to ask what the difference is between massive planets and brown dwarfs. Attempts to answer this question based on for example the mass alone would be problematic, since there appears to be an overlap in mass (Baraffe et al. 2010).

A more fruitful line of discussion is to consider the abundance ratios of heavy elements to hydrogen and helium, especially in comparison to the abundances of the parent stars in the case of planets. Arguably the main physical distinction that can be made between massive planets and very low mass stars is that planets in general are underabundant in light elements, relative to the abundance of the host star. The main attention in this invited contribution is focussed on this difference, and on aspects of planet formation related to it.

It is indeed somewhat odd that, even though it is clear from first principles that the heavy elements residing in rocky planets and in the cores of gas and ice giants were once accompanied by the corresponding amounts of light elements, the issue of when and how the separation between light and heavy elements occurred is largely ignored in current planet formation paradigms. For reasons that are left essentially unexplained it is generally assumed that the protoplanetary disks that eventually built planets, at some convenient 'initial time' consisted of a suitable collection of planetesimals, with a gaseous component whose presence was needed mainly to supply the atmosphere of gas giants, and - when so called for-provide a migration mechanism that can be used to explain exoplanets with otherwise inexplicably small orbital radii.

Increased focus on the actual separation mechanism is not only prudent but may also provide an important background for new ideas about planet formation. Below I discuss some recent ideas that fall in this category and based on the discussion I propose a new planet formation paradigm to encompass these ideas. 


\section{Brown dwarf formation mechanisms}

Star formation occurs (only) in dark and cold molecular clouds. These form by compression of the warm interstellar medium (ISM) due to turbulence, driven by energy input from supernovae (de Avillez \& Breitschwerdt 2005, 2004, 2007) and other mechanisms such as large scale density waves (Shetty \& Ostriker 2008; Kim et al. 2008, 2010). Once a region of the ISM has been compressed enough to become opaque (self-shielding) to the otherwise generally pervasive background UV-light from hot stars it cools very efficiently, until the temperature is stabilized at about $10-20 \mathrm{~K}$, as a balance between heating by cosmic rays and cooling by infra-red radiation. The transition is fast and sharp; essentially a phase transition (McKee \& Ostriker 2007).

In the warm medium the energy densities per unit volume of kinetic energy $\left\langle\rho u^{2} / 2\right\rangle$, thermal energy $\left\langle\rho e_{\text {internal }}\right\rangle$, and magnetic energy $\left\langle B^{2} / 2\right\rangle$ are all similar. However, the sharp drop in temperature when going through the phase transition results in an increase in density (at some constant fiducial gas pressure) by about two orders of magnitude. Thus, while the thermal and magnetic energies after the phase transition initially remain similar, the kinetic energy per unit mass has been greatly increased. The situation is thus one with supersonic and super-Alfvénic turbulence. Indeed, the scenario explains why the situation arises; the turbulence becomes supersonic not because of 'driving', but because the sound speed drops while inertia maintains the velocity magnitude.

Note that, while the pressure of iso-thermal gas increases only linearly with compression magnetic pressure increases as the square of the compression. Because of this the compression ratio and fragment size is often determined by the magnetic pressure rather than by the gas pressure (Padoan \& Nordlund 2002).

Turbulent fragments with masses larger than about a solar mass are essentially always dense enough to collapse by selfgravity, while fragments much smaller than a solar mass are dense enough in only a decreasing fraction (with fragment mass) of cases; this is the reason for the turn-over of the stellar initial mass function at the low mass end (Padoan \& Nordlund 2002). But due to the random nature of turbulence even fragments with masses corresponding to low mass brown dwarfs occasionally reach high enough densities to collapse.

Brown dwarfs are indeed generally believed to form through the mechanism of gravitational instability although there are differences in opinion about details; i.e., whether brown dwarf formation happens in essentially the same manner as normal star formation, if premature abortion of accretion that would otherwise lead to the formation of a more massive ('normal') star is important (Reipurth \& Clarke 2001), or if perhaps there could be a significant contribution due to fragmentation and gravitational collapse in disks around more massive proto-stars followed by ejection from the disk due to gravitational interactions (Stamatellos \& Whitworth 2009). Whether or not such modified formation routes are significant, the main mechanism is essentially the same; gravitational collapse of a fraction of the pre-stellar core material, with unchanged ratios $\mathrm{X}: \mathrm{Y}: \mathrm{Z}$ captured in the brown dwarf, which thus abundance-wise is identical to larger mass stars formed in the same molecular cloud.

The fact the brown dwarf mass stars can form by turbulent fragmentation followed by gravitational collapse in molecular clouds with realistic values of supersonic and super-Alfvénic turbulence has been demonstrated by direct numerical simulations; e.g. by Padoan \& Nordlund (2011). Some of the brown dwarfs created there have accretion histories that are cut short due to interactions and ejections from there natal cores, but that is the case also for normal stars. On the other hand, many brown dwarfs form simply because the fragment they form out of is compressed enough to become gravitationally 
unstable, even though the fragment mass lies in the brown dwarf regime. One concludes, therefore, that even though interactions and ejections play a role they are not necessary for the formation of brown dwarfs.

\section{Planet formation mechanisms}

While the formation of brown dwarfs is reasonably well understood and rests on a firm basis of numerical simulations and corresponding semi-analytical theory the same cannot be said about the theory of planet formation. In the context of massive planet formation there are two complementary scenarios, presumed to perhaps both be applicable, but in different regions of parameter space. The 'core accretion' scenario is widely accepted as being able to explain the formation of Jupiter and Saturn type gas giants, at least under some circumstances, while the 'gravitational instability' scenario is allowed to step in under circumstances where the core accretion scenario has little or no chance of working.

None of these scenarios is entirely convincing, for one thing precisely because none of them is general enough to work under all circumstances, but also for a number of specific reasons briefly mentioned below.

The core accretion scenario has problems creating even Jupiter and Saturn in a time short enough to also allow rocky planets to form without migrating into the Sun (Morishima et al. 2010), and Uranus and Neptune are required to initially form in close proximity to Jupiter and Saturn and to subsequently migrate to their present locations (Tsiganis et al. 2005). While this is in principle a possible work-around in the particular cases of Uranus and Neptune there are many other gas and ice giants discovered in exoplanetary systems, some at considerable distances from their host stars (sometimes of low mass), thus further aggravating the problems with time scales in the core accretion scenario.

Furthermore, in this scenario it is difficult to explain the abundance pattern of Jupiter, which has roughly a factor of three overabundance of most heavy elements, including noble gases (Owen et al. 1999). To achieve such an abundance pattern with core accretion one must rely on clathration of noble gases, a process that takes place only at extremely low temperatures. These ices must then be brought in to accrete onto Jupiter, in exactly the right proportions to reach the same overabundance as much more easily accreted elements. Unless one applies the popular 'Jupiter-is-there-so-it-must-have-happened' argument the explanation must be considered farfetched.

The core accretion scenario also inherits the weaknesses of the general paradigm for rocky planet formation; the meter-size drift barrier (Weidenschilling 1977), the problem of getting pebbles and rocks to grow by sticking rather than braking apart due to collisions, and the general problem of finalizing planet building in a time sufficiently short to prevent Type I migration from being a problem (Morishima et al. 2010).

The gravitational instability (GI) scenario, on the other hand, has a similar list of weaknesses, starting again with the need to invoke a complementary scenario in cases where the GI scenario cannot work. There are severe difficulties in explaining the composition of gas and (particularly) ice giants; their heavily modified abundance patterns can be achieved only be invoking extraordinary strong sources of external radiation to 'boil off' unwanted light element components of the original composition. Another weakness is that the expected dependence on metal abundance is the opposite of the one actually observed; it should be more difficult for the gravitational instability to operate at higher metallicity since larger opacities lead to longer cooling times (Cai et al. 2006). Ironically, the GI scenario seems to mainly survive to step in when the core accretion scenario cannot possibly work. 
As discussed further below the recent discovery of a streaming instability (Youdin \& Goodman 2005), its incorporation into a scenario for very rapid formation of planetesimals (Johansen \& Youdin 2007; Johansen et al. 2007), and the recent extension to a scenario that involves 'pebble accretion' onto large asteroids (Johansen \& Lacerda 2010) are very significant developments, which may turn out to be able to help resolve some of the main issues with the current planet formation scenarios; see also Boley \& Durisen (2010) and Nayakshin (2010, this volume).

\section{Evidence from cosmo-chemistry}

Evidence from isotopic abundances can provide very important information about time scales and thermal histories of the early solar system. Live ${ }^{26} \mathrm{Al}$ and ${ }^{60} \mathrm{Fe}$ (half lives 0.72 Myr and 2.6 Myr, respectively) were present in the early solar system, which allows only a few million years to have passed since their production until the formation of the solar system. After being incorporated in solids, these short-lived radioactive isotopes function as potentially very accurate clocks, in that both parent and daughter nuclei are locked in minerals and differences in their ratios can be recovered today, using high precision mass spectrometers (e.g. Trinquier et al. 2008; Larsen et al. 2010). In addition ${ }^{26} \mathrm{Al}$ was present in sufficient quantities to melt the interior of even rather small asteroids (Baker et al. 2005), and more long lived radioactive isotopes are still important contributors to the internal heat balance of planets.

Combined evidence from several isotopes, particularly ${ }^{54} \mathrm{Cr}$ and ${ }^{26} \mathrm{Mg}$, provide strong evidence for that these isotopes were initially homogeneously distributed, in that the abundance of heavy element enrichment products from different types of stars are strongly correlated in meteorite samples from different parts of the solar system (Thrane et al. 2008). Related evidence indicates that bulk portions of the solar system solids were subsequently subjected to strong thermal processing.

Recent improvements in the precision of ${ }^{26} \mathrm{Al} \rightarrow{ }^{26} \mathrm{Mg}$ analysis indicate, additionally, that the thermal processing caused significant heterogeneity of the ${ }^{26} \mathrm{Al}$ distribution (Larsen et al. 2010). This introduces significant uncertainties in previous age determinations based on the assumption that ${ }^{26} \mathrm{Al}$ was still homogeneously distributed at the time when the first solids formed in the solar system.

However, rather sensationally, the same analysis also shows an internal consistency of the measurements that puts an upper limit of only about 4000 years on the interval of time over which the (asteroid) parent body of the analyzed samples formed. This provides direct evidence for a very rapid planetesimal formation mechanism, consistent with recent astrophysical ideas and empirical evidence (Johansen \& Lacerda 2010; Bottke et al. 2005; Morbidelli et al. 2009).

\section{A new paradigm for planet formation}

The 'streaming instability' mechanism (Johansen \& Youdin 2007; Johansen et al. 2007), and related works (Boley \& Durisen 2010; Nayakshin 2010) have opened up a new and very promising route, which could eventually lead to a much improved understanding of planet formation. One of the most important aspects of these new ideas is that they open up a way to not only avoid the meter size drift barrier (Weidenschilling 1977), but to actually turn the very same mechanism into an important part of planet formation. Johansen \& Lacerda (2010) point out that accretion of cm-size particles ('pebbles') driven by the Weidenschilling (1977) mechanism leads to a natural explanation for a weak but clear tendency of prograde rotation of the largest asteroids. Indeed, one may 
ask "Why stop at planetesimals?"; there seems to be no reason why the rapid growth of planetesimals due to 'pebble accretion' should not continue to build larger bodies, since growing mass leads to a corresponding growth of the Hill sphere, and thus presumably continued rapid accretion. Indeed such a mechanism could also be a natural explanation for the prograde and rapid rotation of a majority of the planets (Nayakshin this volume).

The cosmo-chemical evidence for very short times scales, and for bulk thermal processing at high temperatures in the early solar system also calls for shifting the perspective and focusing on processes involving both dynamical and thermal interaction of gas and solids. Even the structure of the early Sun itself may depend critically on details of the accretion history (Vorobyov \& Basu 2005; Baraffe \& Chabrier 2010; Inutsuka et al. 2010).

These new pieces of evidence and ideas related to the interaction of gas and solids in the early solar system and other proto-planetary systems are components of what could indeed develop into a new paradigm for planet formation. Major issues to be focussed upon in this paradigm are "When and how were heavy elements $(\mathrm{Z})$ separated from the light element components $(\mathrm{X}+\mathrm{Y})$ of the gas?", "What were the main mechanisms responsible for thermal processing of gas and solids?", and "How did the strong mass fractionation that is so evident in the atmospheres of rocky planets happen?".

Whether one thinks the outcome of research along these lines of questioning may change or current picture of planet formation drastically or not, it is certainly important to try to answer these and other questions that may be inspired by such a new paradigm.

\section{Atmospheres of Planets}

As an example where taking the point of view of the new paradigm may turn out to be relevant let's explore the consequences of assuming that 'pebble accretion' is sufficiently efficient to rapidly form rocky cores with planetary masses, embedded in a dense gaseous disk, and that the rocky cores will attract massive gaseous envelopes with temperatures of the order $1500-2000 \mathrm{~K}$.

Such initial states have the interesting property that they evolve, rather independently of details of the initial states, towards rocky planets with atmospheres broadly consistent with the atmospheres that our solar system planets have (or can be surmised to have had). To see this, assume at first that the gaseous atmospheres are so massive that they dominate the total mass inside the systems Hill sphere.

The total optical depth of such an atmosphere is of the order

$$
\tau \sim \kappa M R_{\mathrm{Hill}}^{-2}
$$

where $\kappa$ is the opacity (of the order of $1 \mathrm{~cm}^{2} \mathrm{~g}^{-1}$ or larger for gas+dust at these temperatures). For $M=M_{\text {Earth }}$ the estimated optical depth is of the order of several times $10^{5}$. Such an atmosphere is thus extremely optically thick and evolves, at least on short time scales, nearly adiabatically.

The escape speed becomes formally zero at the Hill sphere, but one can estimate its order of magnitude at radii somewhat smaller than the Hill radius from

$$
v_{\text {escape }}^{2}=\frac{G M}{R_{\text {Hill }}} .
$$

Even for masses as large as $M=10 M_{\text {Earth }}$ the escape speed estimate is less that a thousand meters per second. At $\mathrm{T}=1500 \mathrm{~K}$ the thermal speeds of hydrogen molecules, helium atoms, and nitrogen molecules on the other hand are $\sim 2500,1800$, and $700 \mathrm{~m} \mathrm{~s}^{-1}$, respectively. Hydrogen molecules and helium atoms thus escape easily, via the 
'hydrodynamic escape' mechanism, while nitrogen molecules and other heavy molecules and atoms escape less easily. This would lead to strong mass fractionation, as is indeed observed to have occurred in the Earth's and Mars' atmospheres (Pepin 1991).

The hydrodynamic escape of hydrogen and helium, which in this phase are the main constituents of the atmosphere, leads to a decrease of mass, which in turn leads to an even more rapid escape of the light elements, since the reduction of the Hill radius with mass is more than compensated for by the reduction of the force of gravity. (The mass loss takes place at approximately constant internal entropy, which makes it possible to predict and include also the change in temperature when evaluating the evolution of the balance between gravitational and gas pressure forces.)

The hydrodynamic escape thus continues first of all to the point when the mass of the gaseous envelope becomes smaller than the mass of the rocky core. From that point on the Hill radius remains approximately unchanged, while the atmospheric density continues to decrease by hydrodynamic escape. The decreasing mass density implies a corresponding tendency for an adiabatic temperature decrease, but this is likely to be counteracted by heating from the hot surface of the rocky planet core.

The hydrodynamic escape in the neighborhood of the Hill radius is thus bound to continue until the atmosphere becomes sufficiently transparent to cool efficiently. One can estimate the total mass of the remaining atmosphere by requiring that it has an optical depth of the order of unity if it fills the Hill sphere. From Eq. 6.1 above one concludes that this does not happen until the remaining atmosphere weighs only a fraction of the order of $10^{-6}$ to $10^{-5}$ of the mass of an Earth size rocky planet core. But once the optical depth of the atmosphere becomes small enough the temperature drops and the remaining atmosphere shrinks to a size compatible with the lower temperature, thus effectively shutting down the hydrodynamic escape mechanism.

Mass fractions of $10^{-6}$ to $10^{-5}$ are consistent with the current atmospheres of Earth and Venus (note that the large difference between the atmospheric pressures on Earth and Venus is mainly due to pressure from $\mathrm{CO}_{2}$ while the partial pressures of $\mathrm{N}_{2}$ for example do not differ by more than a factor of a few). The estimate is also consistent with a significantly higher initial atmospheric pressure on Mars than the current one, as required by the evidence for earlier abundant presence of liquid water. However, the escape velocity at the top of Mars atmosphere is so low that even nitrogen and oxygen molecules have thermal speeds where molecules in the tail of the Maxwell distribution can escape, and as evidenced by the very low pressure of the current Martian atmosphere the effect has been sufficient to remove a large fraction of the atmosphere that must once have been present.

The same fate would even more rapidly and efficiently result from any attempt to endow Mercury with an atmosphere, so even if it would have attracted one during the period with a high pressure gaseous proto-planetary disk the current state, with practically no atmosphere, is also consistent with the assumptions made here.

Jupiter, on the other hand, was heavy enough that even if the inial mass was consistent with overall solar abundances (and thus with an initial Jupiter mass about three times larger than now) the estimates of escape speeds and thermal speeds show that at least the outermost layers of an initially hot atmosphere would have been lost. However, already at a radius of about half the Hill radius the escape speed exceeds the likely thermal speeds of hydrogen molecules and helium atoms. This is fully consistent with the fact that Jupiter seems to have retained about $1 / 3$ of an atmosphere with initially solar abundance (a much simpler explanation for Jupiters pattern of abundances of heavy elements than accretion of planetesimals after creation). 
Thus, estimates of the masses of planetary atmospheres based on the assumptions above appear to be fully consistent with current planetary atmospheres (and lack thereof).

\section{Summary and conclusions}

The similarity of the internal structure of giant planets and brown dwarfs and their overlapping mass intervals makes it difficult to distinguish between massive planets and very low mass brown dwarfs. However, in general planets are underabundant in the lightest elements (hydrogen and helium) relative to the primordial composition of the gas that formed their host stars, a fact that must reflect their different mode of formation.

A mechanisms that can separate the gaseous and solid components was discussed by Weidenschilling (1977), but in a context where its consequences actually constituted a major problem for planet formation. Recent developments (Johansen \& Lacerda 2010; Boley \& Durisen 2010; Nayakshin 2010) have made it clear that the mechanism can be turned around into being instead beneficial for planet formation, and the mechanism has already been proven to allow very rapid formation of asteroid-size bodies. Possibly this 'pebble accretion' mechanism could turn out to also be the main mechanism responsible for the formation of rocky planets and giant planet cores.

In the mean time it is already clear that if planet sized rocky cores form in a gaseous proto-planetary disk then Earth sized planets will naturally acquire atmospheres that are essentially devoid of the light elements and that have total masses compatible with the current atmospheres of Earth and Venus. The atmospheres of planets built around smaller rocky cores will suffer heavy atmospheric losses to space, while much more massive cores will be able to hold on to atmospheres compatible with the ones belonging to gas and ice giants.

With such a paradigm water would be a natural and initial part of planetary atmospheres, rather than something that would have to be added to it afterwards via comets or water bearing planetesimals.

In the new paradigm the abundance pattern of Jupiter may be understood as simply reflecting the loss of about $2 / 3$ of the initial amount of light elements, rather than being caused by 'enrichment' of a gaseous atmosphere bombarded by a carefully instrumented collection of rocks and clathrated ices.

The new paradigm also supplies a natural explanation for the heavy mass fractionation of the atmospheres of Venus, Earth and Mars, something that has otherwise been long forgotten and neglected (Pepin 1991).

In conclusion, a renewed focus on the mechanisms that separated solids and gases in the early solar system is of interest by itself, and may in the end turn out to fundamentally change our understanding of planet formation.

\section{References}

Baker, J., Bizzarro, M., Wittig, N., Connelly, J., \& Haack, H. 2005, Nature, 436, 1127

Baraffe, I. \& Chabrier, G. 2010, A\&A, 521, A44+

Baraffe, I., Chabrier, G., Allard, F., \& Hauschildt, P. H. 2002, A\&A, 382, 563

Baraffe, I., Chabrier, G., \& Barman, T. 2010, Reports on Progress in Physics, 73, 016901

Boley, A. C. \& Durisen, R. H. 2010, ApJ, 724, 618

Bottke, W. F., Durda, D. D., Nesvorný, D., Jedicke, R., Morbidelli, A., Vokrouhlický, D., \& Levison, H. 2005, Icarus, 175, 111

Cai, K., Durisen, R. H., Michael, S., Boley, A. C., Mejía, A. C., Pickett, M. K., \& D’Alessio, P. 2006, ApJ (Letters), 636, L149

Chabrier, G., Baraffe, I., Allard, F., \& Hauschildt, P. 2000, ApJ, 542, 464 
Chabrier, G., Baraffe, I., Leconte, J., Gallardo, J., \& Barman, T. 2009, in American Institute of Physics Conference Series, Vol. 1094, American Institute of Physics Conference Series, ed. E. Stempels, 102-111

de Avillez, M. A. \& Breitschwerdt, D. 2004, A\& A, 425, 899

-. 2005, A\&A, 436, 585

-. 2007, ApJ (Letters), 665, L35

Inutsuka, S., Machida, M. N., \& Matsumoto, T. 2010, ApJ (Letters), 718, L58

Johansen, A. \& Lacerda, P. 2010, MNRAS, 404, 475

Johansen, A., Oishi, J. S., Mac Low, M., Klahr, H., Henning, T., \& Youdin, A. 2007, Nature, 448,1022

Johansen, A. \& Youdin, A. 2007, ApJ, 662, 627

Kim, C., Kim, W., \& Ostriker, E. C. 2008, ApJ, 681, 1148

—. 2010, ApJ, 720, 1454

Larsen, K., Trinquier, A., Paton, C., Ivanova, M., Nordlund, A., Krot, A. N., \& Bizzarro, M. 2010, Meteoritics and Planetary Science Supplement, 73, 5202

McKee, C. F. \& Ostriker, E. C. 2007, ARA $\& A$, 45, 565

Morbidelli, A., Bottke, W. F., Nesvorný, D., \& Levison, H. F. 2009, Icarus, 204, 558

Morishima, R., Stadel, J., \& Moore, B. 2010, Icarus, 207, 517

Nayakshin, S. 2010, MNRAS, 408, L36

Nayakshin, S. 2011, this volume

Owen, T., Mahaffy, P., Niemann, H. B., Atreya, S., Donahue, T., Bar-Nun, A., \& de Pater, I. 1999, Nature, 402, 269

Padoan, P. \& Nordlund, Å. 2002, ApJ, 576, 870

Padoan, P. \& Nordlund, A. 2011, ApJ, 730, id.40

Pepin, R. O. 1991, Icarus, 92, 2

Reipurth, B. \& Clarke, C. 2001, AJ, 122, 432

Shetty, R. \& Ostriker, E. C. 2008, ApJ, 684, 978

Stamatellos, D. \& Whitworth, A. P. 2009, MNRAS, 400, 1563

Thrane, K., Nagashima, K., Krot, A. N., \& Bizzarro, M. 2008, ApJ (Letters), 680, L141

Trinquier, A., Bizzarro, M., Ulfbeck, D., Elliott, T., Coath, C. D., Mendybaev, R. A., Richter, F. M., \& Krot, A. N. 2008, Geochimica et Cosmochimica Acta Supplement, 72, 956

Tsiganis, K., Gomes, R., Morbidelli, A., \& Levison, H. F. 2005, Nature, 435, 459

Vorobyov, E. I. \& Basu, S. 2005, ApJ (Letters), 633, L137

Weidenschilling, S. J. 1977, MNRAS, 180, 57

Youdin, A. N. \& Goodman, J. 2005, ApJ, 620, 459 